\title{
Cellular structure for the Herzog-Takayama resolution
}

\author{
Afshin Goodarzi
}

Received: 18 May 2013 / Accepted: 7 April 2014 / Published online: 24 April 2014

(C) Springer Science+Business Media New York 2014

\begin{abstract}
Herzog and Takayama constructed an explicit resolution for the ideals with a regular linear quotient. These ideals include all matroidal and stable ideals. The resolutions of matroidal and stable ideals are known to be cellular. In this note, we show that the Herzog-Takayama resolution is also cellular.
\end{abstract}

Keywords Cellular resolution - Monomial ideals · Linear quotient · Convex geometry

\section{Introduction}

In this section we describe the problem. Precise definitions will appear in the following sections. For undefined terminology we refer to the books by Hatcher [8], Peeva [15] and Miller and Sturmfels [13]. The idea of determining free resolutions of monomial ideals in a polynomial ring using labelled chain complexes of topological objects was introduced by Bayer et al. [2] by considering the labelled chain complex of simplicial complexes. Every monomial ideal admits such a resolution, namely the Taylor resolution, although most of the time it is far from being minimal. This method was generalized in [3], where the authors constructed a regular cell complex (the Hull complex) for any monomial ideal. From its labelled chain complex one gets a resolution of the ideal which in general is much shorter than the Taylor resolution. Batzies and Welker in [1] developed an algebraic analogue of Forman's discrete Morse theory to minimize the resolutions.

Unfortunately, even if we consider the more general case of CW complexes, as the authors did in [1], it is not the case that one can describe the minimal free resolution

\footnotetext{
A. Goodarzi $(\varangle)$

Department of Mathematics, Royal Institute of Technology, 10044 Stockholm, Sweden

e-mail: af.goodarzi@gmail.com; afshingo@kth.se
} 
of a monomial ideal using these methods. A family of non-examples is given in [18]. However, for a monomial ideal with a linear quotient, it is shown in [1, Proposition 4.3] that there exists a CW-complex which supports the minimal free resolution of the ideal. The minimal free resolution of a class of ideals with a linear quotient, the so called ideals with a regular linear quotient (see Definition 2.3), is determined by Herzog and Takayama in [9]. This class contains all matroidal ideals, stable ideals and square-free stable ideals. In [14], the authors have given a polytopal complex that supports the minimal free resolution of matroidal ideals. In [12], Mermin constructed a regular cell complex that supports the Eliahou-Kervaire resolution for stable ideals. On the other hand, since the square-free part of the Eliahou-Kervaire resolution resolves square-free stable ideals minimally, such a construction for square-free stable ideals exists. So it is natural to ask whether the Herzog-Takayama resolution is cellular or not. The aim of this paper is to give a positive answer to the question above by explicitly constructing a regular cell complex that supports the Herzog-Takayama resolution for ideals with a regular linear quotient.

\section{Monomial ideals with a linear quotient and cellular resolution}

Let $K$ be a field and $S=K\left[x_{1}, \ldots, x_{n}\right]$ be the polynomial ring in $n$ variables over $K$. For a monomial ideal $I$ in $S$, we denote by $M(I)$ the set of all monomials in $I$. We also denote by $G(I)$ the unique minimal set of generators of $I$. We say that $I$ has a linear quotient, if $G(I)$ admits an admissible order, that is a linear ordering $u_{1}, \ldots, u_{m}$ of monomials in $G(I)$ such that the colon ideal $\left\langle u_{1}, \ldots, u_{j-1}\right\rangle: u_{j}$ is generated by a subset $\mathfrak{q}\left(u_{j}\right)$ of variables for all $2 \leq j \leq m$. To any admissible order of $I$, one can associate a unique decomposition function, that is, a function $\mathfrak{g}: M(I) \rightarrow G(I)$ that maps a monomial $v$ to $u_{j}$, if $j$ is the smallest index for which $v \in I_{j}$, where $I_{j}:=\left\langle u_{1}, \ldots, u_{j}\right\rangle$ (see [9] for more on decomposition functions).

It is not difficult to check that an ordering $u_{1}, \ldots, u_{m}$ of $G(I)$ is an admissible order if and only if it satisfies the following shelling type condition, considered by Batzies and Welker [1, p. 157]:

$$
\begin{aligned}
& \text { For all } j \text { and } i<j \text { there exists } k<j \text { such that } \\
& \operatorname{lcm}\left(u_{k}, u_{j}\right)=x_{t} \cdot u_{j} \text { for some } x_{t} \text { and } \operatorname{lcm}\left(u_{k}, u_{j}\right) \\
& \text { divides } \operatorname{lcm}\left(u_{i}, u_{j}\right) .
\end{aligned}
$$

It is known [10, Lemma 2.1] that for a monomial ideal with a linear quotient there always exists a degree increasing admissible order. So, throughout this note all admissible orders are considered to be increasing.

The following result was proved in [1, Proposition 4.3] by using algebraic discrete Morse theory. Here, we give an alternative proof based on the observation that the resolutions obtained by iterated mapping cones are supported by $\mathrm{CW}$ complexes that can be constructed by iterated (topological) mapping cones. This is indeed the idea behind our construction in the next section. 
Proposition 2.1 For an ideal I with a linear quotient, there exists a $C W$-complex $X_{I}$ that supports the minimal free resolution of $S / I$.

Proof Let $u_{1}, \ldots, u_{m}$ be a degree increasing admissible order of $G(I)$. Assume that we have inductively constructed a $\mathrm{CW}$-complex $X_{j-1}$ that supports the minimal free resolution of $S / I_{j-1}$. Also, let $\Delta$ be the simplex associated to the Koszul resolution of $S /\left\langle\mathfrak{q}\left(u_{j}\right)\right\rangle$. Lifting the left non-zero map of the short exact sequence

$$
0 \longrightarrow S /\left\langle\mathfrak{q}\left(u_{j}\right)\right\rangle \longrightarrow S / I_{j-1} \longrightarrow S / I_{j} \longrightarrow 0
$$

to the minimal free resolutions of $S /\left\langle\mathfrak{q}\left(u_{j}\right)\right\rangle$ and $S / I_{j-1}$, then induces a cellular map of $\Delta$ into $X_{j-1}$. Therefore, the resolution of $S / I_{j}$ obtained by the homological mapping cone has a cellular structure that is the topological mapping cone of the cellular map of $\Delta$ into $X_{j-1}$. The fact that this resolution is minimal follows from [9, Lemma 1.5]

Remark 2.2 Reiner and Welker [16, Sect. 5] gave an example of an ideal with a linear quotient for which the differential matrices in the minimal free resolution cannot be written using only \pm 1 coefficients. This shows that the cell complex $X_{I}$ in Proposition 2.1 is not regular in general.

The difficulty of constructing the cell complex $X_{I}$ in practice is then how to define the cellular maps in each step, which is indeed as difficult as defining the comparison maps $S /\left\langle\mathfrak{q}\left(u_{j}\right)\right\rangle \rightarrow S / I_{j-1}$. This has been already observed by Herzog and Takayama in [9], where the authors defined a subfamily of ideals with a linear quotient that have decomposition function with a similar behaviour as of the stable ideals'.

Definition 2.3 A decomposition function $\mathfrak{g}$ is said to be regular, if $\mathfrak{q}\left(\mathfrak{g}\left(y u_{j}\right)\right) \subseteq \mathfrak{q}\left(u_{j}\right)$, for any $j$ and any $y \in \mathfrak{q}\left(u_{j}\right)$. We say that $I$ has a regular linear quotient, if it admits an admissible order with a regular decomposition function.

We will frequently use the following equation for the decomposition function of ideals having regular linear quotient, proved in [9]:

$$
\mathfrak{g}(y \mathfrak{g}(z u))=\mathfrak{g}(z \mathfrak{g}(y u)) \quad \forall u \in G(I) \quad \forall z, y \in \mathfrak{q}(u) .
$$

It can be easily checked that the set of ideals with a regular linear quotient contains all stable and square-free stable ideals. On the other hand, it is known [9, Theorem 1.10] that the reverse lexicographic order on minimal generators of a matroidal ideal is an admissible order with a regular decomposition function.

Definition 2.4 Let $\sigma$ be a subset of $V=\left\{x_{1}, \ldots, x_{n}\right\}$ and assume the total ordering $x_{1}<\cdots<x_{n}$ on $V$. Then for an element $y \in \sigma$ set $\alpha(\sigma ; u)$ to be the number of variables $z$ in $\sigma$ such that $z<y$.

Construction 2.5 Let $I$ be a monomial ideal with a regular linear quotient. The Herzog-Takayama resolution $\mathbf{F}_{I}$ of $S / I$ has basis denoted

$$
\mathcal{B}=\{1\} \cup\{f(\sigma ; u) \mid u \in G(I) \text { and } \sigma \subseteq \mathfrak{q}(u)\} \text {, }
$$


where the element $f(\sigma ; u)$ has homological degree $|\sigma|+1$. If $\sigma$ is non-empty, then we define

$$
\mu(f(\sigma ; u))=\sum_{y \in \sigma}(-1)^{\alpha(\sigma ; u)} y f(\sigma-\{y\} ; u),
$$

and

$$
\delta(f(\sigma ; u))=\sum_{y \in \sigma}(-1)^{\alpha(\sigma ; u)} \frac{y u}{\mathfrak{g}(y u)} f(\sigma-\{y\} ; \mathfrak{g}(y u)),
$$

with the convention that $f(\tau ; v)=0$, if $\tau$ is not a subset of $\mathfrak{q}(v)$. The differential in $\mathbf{F}_{I}$ is given by $\partial=\delta-\mu$, in homological degrees $>1$ and otherwise $\partial(f(\emptyset, u))=u$.

Theorem 2.6 [9, Theorem 1.12] Let I be a monomial ideal with a regular linear quotient. Then the Herzog-Takayama resolution $\mathbf{F}_{I}$ is the minimal free resolution of $S / I$.

\section{Monomial ideals with a regular linear quotient and their associated regular cell complexes}

We now associate to an ideal with a regular linear quotient a regular cell complex as follows:

Construction 3.1 Let $I$ be a monomial ideal with a regular linear quotient with respect to the admissible order $u_{1}, \ldots, u_{m}$ of $G(I)$. Also let $\mathfrak{g}$ be its decomposition function. We will construct a regular labelled cell complex $X_{I}$ inductively as follows:

(i) Let $X_{1}$ be the 0 -simplex with the labelled vertex $\left\{u_{1}\right\}$,

(ii) assume that the regular labelled cell complex $X_{j-1}$ with vertices $u_{1}, \ldots, u_{j-1}$ is constructed.

For what follows, let $u=u_{j}$ be a point outside $X_{j-1}$ and for a subset $\sigma$ of $\mathfrak{q}(u)$, define $\mathfrak{g}(\sigma ; u):=\mathfrak{g}\left(u \cdot \prod_{y \in \sigma} y\right)$. Also, denote by $X(u)$ the subcomplex of $X_{j-1}$, induced by $\{\mathfrak{g}(\sigma ; u)\}$ for all non-empty subsets $\sigma$ of $\mathfrak{q}(u)$. We will show that $X(u)$ is an $(l-1)$-dimensional ball in Lemma 3.4 and Proposition 3.6 below, where $l=|\mathfrak{q}(u)|$.

(iii) glue an $l$-ball $B(u)$ along its boundary to $X(u) \cup(\{u\} * \partial X(u))$.

Having defined the new maximal cell $B(u)$, what remains to have the complete description of $X_{j}$ is to give a cell decomposition for $\{u\} * \partial X(u)$. For this purpose, let us, for any proper subset $\sigma$ of $\mathfrak{q}(u)$, denote by $X(\sigma, u)$ the subcomplex of $X_{j-1}$ induced by the vertices $\{\mathfrak{g}(\tau ; u)\}$ for all non-empty subsets $\tau$ of $\sigma$. The fact that $X(\sigma, u)$ is a ball of dimension $|\sigma|-1$ is needed for the next step, we will discuss it in the end of this section in Remark 3.8.

(iv) define $X_{j}$ to be $X_{j-1} \cup\{B(u)\} \bigcup_{\sigma \subset \mathfrak{q}(u)}\{\{u\} * X(\sigma, u)\}$. 
Remark 3.2 For a non-empty subset $A$ of $G(I)$, the following are equivalent.

(i) There is a cell of $X_{I}$ whose set of vertices coincides with $A$.

(ii) There is some $u \in G(I)$ and some $\sigma \subseteq \mathfrak{q}(u)$ such that

$$
A=\{\mathfrak{g}(\tau ; u) \mid \tau \subseteq \sigma\}
$$

Moreover, this cell corresponds to the base element $f(\sigma ; u)$ of the Herzog-Takayama resolution.

We now study some combinatorial properties of the cell complex $X_{I}$ constructed in 3.1, via a simplicial subdivision of it. We follow the notation in Construction 3.1. Let us start by giving the simplicial subdivision of $X_{I}$.

Construction 3.3 We construct a simplicial complex $\Lambda_{I}$ inductively as follows:

(i) Let $\Lambda_{1}$ be the 0 -simplex $\left\{u_{1}\right\}$,

(ii) Assume that $\Lambda_{j-1}$ is constructed,

(iii) Take a cone with apex $\{u\}$ over the subcomplex $\Lambda(u)$ of $\Lambda_{j-1}$, induced by $\{\mathfrak{g}(\sigma ; u)\}$, for all subsets $\sigma$ of $\mathfrak{q}(u)$, to obtain

$$
\Lambda_{j}=\Lambda_{j-1} \bigcup(\{u\} * \Lambda(u))
$$

The following is immediate.

Lemma 3.4 $\Lambda_{j}$ (respectively $\Lambda(u)$ ) is a simplicial subdivision of $X_{j}$ (respectively $X(u))$.

A closure operator on a finite set $E$ is a function $c: 2^{E} \rightarrow 2^{E}$, such that for all $\sigma, \tau \subseteq E$,

(CO1) $\sigma \subseteq c(\sigma)$,

(CO2) $\sigma \subseteq \tau$ implies $c(\sigma) \subseteq c(\tau)$,

$(\mathrm{CO} 3) c(c(\sigma))=c(\sigma)$.

A closure operator $c$ is said to be an anti-exchange closure if, in addition, for all $a \neq b$ in $E$, it satisfies the following anti-exchange axiom:

(AE) If $a, b \notin c(\sigma)$ and $a \in c(\{b\} \cup \sigma)$, then $b \notin c(\{a\} \cup \sigma)$.

A finite set $E$ together with an anti-exchange closure $c$ on it is called a convex geometry. The reader may consult Björner and Ziegler [6], for a comprehensive introduction to a more general topic, greedoids. Now let us define a closure operator on $\mathfrak{q}(u)$ : For a subset $\sigma$ of $\mathfrak{q}(u)$, we let $c(\sigma)$ to be the largest subset $\tau$ of $\mathfrak{q}(u)$ such that $\mathfrak{g}(\sigma ; u)=\mathfrak{g}(\tau ; u)$. The fact that this operator is well defined follows from the simple observation that for any $\delta_{1}$ and $\delta_{2}$ if $\mathfrak{g}\left(\delta_{1} ; u\right)=\mathfrak{g}\left(\delta_{2} ; u\right)$, then $\mathfrak{g}\left(\delta_{1} ; u\right)=\mathfrak{g}\left(\delta_{1} \cup \delta_{2} ; u\right)$.

Proposition 3.5 The pair $\langle\mathfrak{q}(u), c\rangle$ is a convex geometry. 
Proof The axioms (CO1) and (CO3) clearly hold. Assume that $\tau$ is the union $\delta \cup \sigma$. We have

$$
\mathfrak{g}(\delta \cup \sigma ; u)=\mathfrak{g}(\delta ; \mathfrak{g}(\sigma ; u))=\mathfrak{g}(\delta ; \mathfrak{g}(c(\sigma) ; u))=\mathfrak{g}(\delta \cup c(\sigma) ; u)
$$

Hence $c(\sigma) \subseteq c(\delta \cup \sigma)=c(\tau)$. Thus, $c$ is a closure operator.

Now, we shall verify the anti-exchange axiom. Let $\sigma$ be any subset of $\mathfrak{q}(u)$ and denote by $v$ the monomial minimal generator $\mathfrak{g}(\sigma ; u)$. The condition that $a \notin c(\sigma)$ is equivalent to $a \in \mathfrak{q}(v)$. Now for a variable $b$ in $\mathfrak{q}(v)$ different from $a$, let $a \in c(\{b\} \cup \sigma)$, or equivalently $\mathfrak{g}(a b v)=\mathfrak{g}(b v)$. The fact that $\mathfrak{g}(a v)$ is a minimal monomial generator different from $v$ that divides $a v$ implies that $\mathfrak{g}(a v) \neq \mathfrak{g}(b v)=\mathfrak{g}(a b v)$ and therefore $b \notin c(\{a\} \cup \sigma)$.

The set of all closed sets of a convex geometry, i.e. those subsets that are fixed under the closure operator, forms a lattice when the partial ordering is inclusion. This lattice is known to be meet-distributive, see e.g. [7, Theorem 3.3].

Proposition 3.6 $\Lambda(u)$ is a shellable $(l-1)$-dimensional ball.

Proof Let $L$ be the lattice of closed sets of $\langle\mathfrak{q}(u), c\rangle$ and $\widehat{L}=L \backslash\{\emptyset\}$. The decomposition function $\mathfrak{g}(. ; u)$ can be seen as a bijection between the elements of $\widehat{L}$ and vertices of $\Lambda(u)$, that maps every closed set $\sigma$ to $\mathfrak{g}(\sigma ; u)$. Furthermore, via this map the faces of $\Lambda(u)$ are precisely chains in $\widehat{L}$ and hence $\Lambda(u)$ is the order complex of $\widehat{L}$. Now, since the order complex of the proper part of any meet-distributive lattice is a shellable ball (see, e.g. [4]), $\Lambda(u)$ is a shellable ball. The argument about dimension follows from [6, Proposition 8.7.2 (iv)].

A simplicial complex $K$ is said to be a $P L-$ ball if a subdivision of $K$ is a subdivision of a simplex. A regular cell complex $\Gamma$ is a PL-ball if its barycentric subdivision is a PL-ball. Many properties that we expect balls to have fail when they are not PL, for instance the complex obtained by gluing two balls with the same dimension along a common codimension one ball lying on their boundaries is not necessarily a ball, if they are not PL. We refer to [5, Sect. 4.7d] and the references therein for a detailed discussion on the subject.

We mentioned that for our construction to be well-defined we need $X(u)$ to be a ball, but actually the inductive construction requires more:

Corollary $3.7 X(u)$ is an $(l-1)$-dimensional $P L-$ ball.

Proof It follows from Lemma 3.4, Proposition 3.6 and [4, Corollary 2.2].

Remark 3.8 Let $\sigma$ be a fixed subset of $\mathfrak{q}(u)$. For a subset $\delta$ of $\sigma$ define $c_{\sigma}(\delta)$ to be the largest subset $\tau$ of $\sigma$ such that $\mathfrak{g}(\delta ; u)=\mathfrak{g}(\tau ; u)$. Then, in a similar way as in Proposition 3.5, one can show that $\left\langle\sigma, c_{\sigma}\right\rangle$ is a convex geometry and the same line of reasoning as we used to prove the above corollary implies that $X(\sigma, u)$ is a PL-ball of dimension $|\sigma|-1$. 


\section{Main result}

In this section, we prove our main result. We begin by proving two auxiliary lemmas.

Lemma 4.1 $X_{I}$ is contractible.

Proof Let $f: X(u) \hookrightarrow X_{j-1}$ be the inclusion map. Then $X_{j}$ is homotopy equivalent to the mapping cone $C_{f}$ of $f$. Now since $X(u)$ and $X_{j-1}$ are contractible, by [17, p. 27] $f$ is a homotopy equivalence and therefore $C_{f}$ is contractible (see, e.g. [8, Sect. 4.2] for more details).

Let $I \subset S$ be a monomial ideal and $\mu$ a monomial in $S$. Then we denote by $I_{\leq \mu}$ the ideal generated by those monomials in $G(I)$ that divide $\mu$.

Lemma 4.2 Let I $\subset S$ be a monomial ideal with linear quotient and let $\mu$ be a monomial in $S$. Then $I_{\leq \mu}$ has linear quotient. Moreover if I is regular, then so is $I_{\leq \mu}$.

Proof Let $u_{1}, \ldots, u_{m}$ be an admissible order of minimal generators of $I$ and assume that $u_{i_{1}}, \ldots, u_{i_{t}}$ generates $I_{\leq \mu}$, where $i_{1}<\cdots<i_{t}$. We show that $u_{i_{1}}, \ldots, u_{i_{t}}$ is an admissible order for $I_{\leq \mu}$. For $s<t$, the shelling type condition 1, guarantees that there exists $l<i_{t} \operatorname{such}$ that $\operatorname{lcm}\left(u_{l}, u_{i_{t}}\right)=u_{i_{t}} x_{r}$ for some $x_{r}$ and that $\operatorname{lcm}\left(u_{l}, u_{i_{t}}\right)$ divides $\operatorname{lcm}\left(u_{i_{s}}, u_{i_{t}}\right)$. In particular $u_{l}$ divides $\mu$ and hence $u_{l} \in I_{\leq \mu}$.

For the second part denote by $\mathfrak{g}^{\prime}$, the decomposition function of $I_{\leq \mu}$. Let $y$ be a variable such that $\mathfrak{g}^{\prime}\left(y u_{i_{j}}\right) \neq u_{i_{j}}$ and $z$ another variable such that $\mathfrak{g}^{\prime}\left(z \mathfrak{g}^{\prime}\left(y u_{i_{j}}\right)\right) \neq \mathfrak{g}^{\prime}\left(y u_{i_{j}}\right)$. To show that $\mathfrak{g}^{\prime}$ is regular, then it is enough to show that $\mathfrak{g}^{\prime}\left(z u_{i_{j}}\right) \neq u_{i_{j}}$. Assume not. Then for any $l<i_{j}$ that $u_{l}$ divides $z u_{i_{j}}, u_{l}$ does not divide $\mu$ and in particular $\operatorname{deg}_{z}\left(u_{i_{j}}\right)=\operatorname{deg}_{z}(\mu)$, where $\operatorname{deg}_{z}$ stands for the degree of $z$ in the monomial. On the other hand, $v=\mathfrak{g}^{\prime}\left(z \mathfrak{g}^{\prime}\left(y u_{i_{j}}\right)\right)$ divides $z y u_{i_{j}}$. So, $v$ divides $y u_{i_{j}}$, since $\operatorname{deg}_{z}(v) \leq$ $\operatorname{deg}_{z}(\mu)=\operatorname{deg}_{z}\left(u_{i_{j}}\right)$. This is a contradiction, since $v=\mathfrak{g}^{\prime}\left(z \mathfrak{g}^{\prime}\left(y u_{i_{j}}\right)\right)$ appears earlier than $\mathfrak{g}^{\prime}\left(y u_{i_{j}}\right)$ in the admissible order.

Now, we are in the position to prove our main result.

Theorem 4.3 If I has a regular linear quotient, then the labelled regular cell complex $X_{I}$ supports the minimal free resolution of $I$.

Proof First observe that for any monomial $\mu$, the subcomplex $X_{\leq \mu}$ of $X_{I}$, consisting of all cells with a label that divides $\mu$, is the same as the complex $X_{I_{\leq \mu}}$ and hence is contractible by Lemmas 4.1 and 4.2.

Now we shall show that any two cells with a non-trivial containment relation have different labels. Let $c$ be a cell of $X_{I}$. Then the vertices of $c$ are $v$ together with $\{\mathfrak{g}(\sigma ; v)\}_{\sigma \subseteq \tau}$, for some $v$ and some $\tau \subseteq \mathfrak{q}(v)$ and in particular the label of $c$ is $v \prod_{z \in \tau} z$. For a maximal cell $c^{\prime}$ of $c$, we have only two possibilities: either $c^{\prime}$ contains $v$ or not. In the former case, the other vertices of $c^{\prime}$ are $\{\mathfrak{g}(\sigma ; v)\}_{\sigma \subseteq \tau^{\prime}}$, where $\tau^{\prime}=\tau \backslash\{y\}$ for some variable $y$. So, the label of $c^{\prime}$ is different from the label of $c$. In the latter case, the vertices of $c^{\prime}$ are $\mathfrak{g}(y v)$ together with $\{\mathfrak{g}(\sigma ; \mathfrak{g}(y v))\}_{\sigma \subseteq \tau^{\prime}}$, for some $y \in \mathfrak{q}(v)$, where $\tau^{\prime}=\tau \backslash\{y\}$. The label of $c^{\prime}$ is then $\mathfrak{g}(y v) \prod_{z \in \tau^{\prime}} z$ which is different from the label of $c$, since $\operatorname{deg}(\mathfrak{g}(y v)) \leq \operatorname{deg}(v)$ and $\left|\tau^{\prime}\right|<|\tau|$. 
Now, considering the correspondence mentioned in Remark 3.2, it is not difficult to see that the signs in the differential in $\mathbf{F}_{I} 2.5$ produce an incidence function on $X_{I}$. Thus, we may conclude that:

Theorem 4.4 Let I be an ideal with a regular linear quotient. Then the labelled regular cell complex $X_{I}$ supports the Herzog-Takayama resolution $\mathbf{F}_{I}$ of $S / I$.

Open problem Lyubeznik [11] presented a method to reduce the Taylor resolution of a monomial ideal. It can be shown that Lyubeznik's resolution is supported on a simplicial complex obtained from a simplex (supporting the Taylor resolution) by a sequence of collapses. Batzies and Welker generalized Lyubeznik's construction using ideas from discrete Morse theory. They applied this construction to show that for a monomial ideal $I$ with a linear quotient there exists a CW-complex that supports the minimal free resolution of $I$ [1, Proposition 4.3]. When $I$ has a regular linear quotient, it would be interesting to determine whether our complex $X_{I}$ necessarily coincides with the one coming from generalized Lyubeznik-resolution (in the sense of [1]) or not.

Acknowledgments I would like to thank Anders Björner for helpful discussions, especially for bringing convex geometries to my attention, Volkmar Welker for some very helpful comments. I would also like to thank the anonymous referees for many helpful suggestions that led to improvements of the exposition.

\section{References}

1. Batzies, E., Welker, V.: Discrete Morse theory for cellular resolutions. J. Reine Angew. Math. 543, 147-168 (2002)

2. Bayer, D., Peeva, I., Sturmfels, B.: Monomial resolutions. Math. Res. Lett. 5, 31-46 (1998)

3. Bayer, D., Sturmfels, B.: Cellular resolutions of monomial modules. J. Reine Angew. Math. 502, 123-140 (1998)

4. Billera, L.J., Hsiao, S.K., Provan, J.S.: Enumeration in convex geometries and associated polytopal subdivisions of spheres. Discret. Comput. Geom. 39, 123-137 (2008)

5. Björner, A., Las Vergnas, M., Sturmfels, B., White, N., Ziegler, G.M.: Oriented Matroids, 2nd edn. Cambridge University Press, Cambridge (1999)

6. Björner, A., Ziegler, G.M.: Introduction to greedoids. In: White, N. (ed.) Matroid Applications, pp. 284-357. Cambridge University Press, Cambridge (1992)

7. Edelman, P.H.: Meet-distributive lattices and the anti-exchange closure. Algebra Univers. 10, 290-299 (1980)

8. Hatcher, A.: Algebraic Topology. Cambridge University Press, Cambridge (2002)

9. Herzog, J., Takayama, Y.: Resolutions by mapping cones. Homology Homotopy Appl. 4, 277-294 (2004)

10. Jahan, A.S., Zheng, X.: Ideals with linear quotients. J. Combin. Theory. Ser. A. 117(1), 104-110 (2010)

11. Lyubeznik, G.: A new explicit finite free resolution of ideals generated by monomials in an $R$-sequence. J. Pure Appl. Alg. 51, 193-195 (1988)

12. Mermin, J.: The Eliahou-Kervaire resolution is cellular. J. Commut. Algebra 2(1), 55-78 (2010)

13. Miller, E., Sturmfels, B.: Combinatorial Commutative Algebra, vol. 227. Springer, New York (2005)

14. Novik, I., Postnikov, A., Sturmfels, B.: Syzygies of oriented matroids. Duke Math. J. 111(2), 287-317 (2002)

15. Peeva, I.: Graded Syzygies. Springer, New York (2010)

16. Reiner, V., Welker, V.: Linear syzygies of Stanley-Reisner ideals. Math. Scand. 89(1), 117-132 (2001)

17. Spanier, E.H.: Algebraic Topology. Springer, New York (1981)

18. Velasco, M.: Minimal free resolutions that are not supported on a CW-complex. J. Algebra 319(1), 102-114 (2008) 